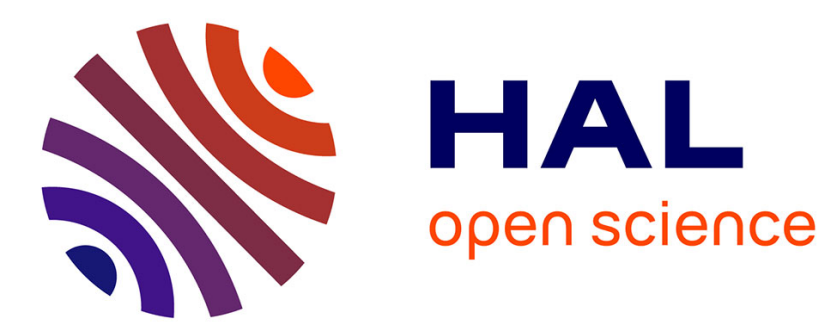

\title{
Isotropically and Anisotropically Weighted Sobolev Spaces for the Oseen Equation
}

Chérif Amrouche, Ulrich Razafison

\section{To cite this version:}

Chérif Amrouche, Ulrich Razafison. Isotropically and Anisotropically Weighted Sobolev Spaces for the Oseen Equation. Advances in Mathematical Fluid Mechanics, 2010, pp.1-24. 10.1007/978-3-64204068-9_1. hal-00335163

\section{HAL Id: hal-00335163 https://hal.science/hal-00335163}

Submitted on 28 Oct 2008

HAL is a multi-disciplinary open access archive for the deposit and dissemination of scientific research documents, whether they are published or not. The documents may come from teaching and research institutions in France or abroad, or from public or private research centers.
L'archive ouverte pluridisciplinaire HAL, est destinée au dépôt et à la diffusion de documents scientifiques de niveau recherche, publiés ou non, émanant des établissements d'enseignement et de recherche français ou étrangers, des laboratoires publics ou privés. 


\title{
Isotropically and Anisotropically Weighted Sobolev Spaces for the Oseen Equation
}

\author{
Chérif Amrouche and Ulrich Razafison \\ Dedicated to Giovanni Paolo Galdi at the occasion of his 60th Birthday
}

\begin{abstract}
This contribution is devoted to the Oseen equations, a linearized form of the Navier-Stokes equations. We give here some results concerning the scalar Oseen operator and we prove Hardy inequalities concerning functions in Sobolev spaces with anisotropic weights that appear in the investigation of the Oseen equations.
\end{abstract}

Mathematics Subject Classification (2000). 76D05, 35Q30, 26D15, 46D35.

Keywords. Oseen equations, anisotropic weights, Hardy inequality, Sobolev weighted spaces.

\section{Introduction}

In an exterior domain $\Omega$ of $\mathbb{R}^{3}$, the Oseen system is obtained by linearizing the Navier-Stokes equations, describing the flow of a viscous fluid past the obstacle $\mathbb{R}^{3} \backslash \bar{\Omega}$, around a nonzero constant vector which is the velocity at infinity. When $\Omega=\mathbb{R}^{3}$, the system can be written as follow:

$$
\begin{aligned}
-\nu \Delta \mathbf{u}+k \frac{\partial \mathbf{u}}{\partial x_{1}}+\nabla \pi=\mathbf{f} & \text { in } \mathbb{R}^{3}, \\
\operatorname{div} \mathbf{u}=g & \text { in } \mathbb{R}^{3},
\end{aligned}
$$

where we add the condition at infinity

$$
\lim _{|\mathbf{x}| \rightarrow \infty} \mathbf{u}(\mathbf{x})=\mathbf{u}_{\infty}
$$

The data are the viscosity of the fluid $\nu$, the external forces acting on the fluid $\mathbf{f}, \mathbf{a}$ function $g$, a constant vector $\mathbf{u}_{\infty}$ and a real $k>0$. The unknowns are the velocity 
of the fluid $\mathbf{u}$ and the pressure function $\pi$. Let us now notice that the pressure satisfies the Laplace equation

$$
\Delta \pi=\operatorname{div} \mathbf{f}+\nu \Delta g-k \frac{\partial g}{\partial x_{1}},
$$

and each component $u_{i}$ of the velocity satisfies

$$
-\nu \Delta u_{i}+k \frac{\partial u_{i}}{\partial x_{1}}=f_{i}-\frac{\partial \pi}{\partial x_{i}} .
$$

Hence we see that the Oseen problem (1.1) is related to the following equation:

$$
-\nu \Delta u+k \frac{\partial u}{\partial x_{1}}=f \text { in } \mathbb{R}^{3} .
$$

Therefore, the results arising from the analysis of (1.5) can be used for the investigation of the Oseen problem (1.1). To prescribe the growth or the decay properties of functions at infinity, we consider here weighted Sobolev spaces where the weight reflects the decay properties of the fundamental solution $\mathcal{O}$ of (1.5) defined by

$$
\mathcal{O}(\mathbf{x})=\frac{1}{4 \pi \nu|\mathbf{x}|} e^{-k\left(|\mathbf{x}|-x_{1}\right) / 2 \nu}
$$

Note now that, at infnity, $\mathcal{O}$ has the same following decay properties than the fundamental solution of Oseen

$$
\mathcal{O}(\mathbf{x})=O\left(\eta_{-1}^{-1}(\mathbf{x})\right), \quad \nabla \mathcal{O}(\mathbf{x})=O\left(\eta_{-3 / 2}^{-3 / 2}(\mathbf{x})\right), \quad \partial^{2} \mathcal{O}(\mathbf{x})=O\left(\eta_{-2}^{-2}(\mathbf{x})\right), \ldots
$$

where $\eta_{\beta}^{\alpha}(\mathbf{x}) \equiv \eta_{\beta}^{\alpha}=(1+|\mathbf{x}|)^{\alpha}\left(1+|\mathbf{x}|-x_{1}\right)^{\beta}$ will be the weight function considered. Equation (1.5) has been investigated by Farwig (see[6]) in weighted $L^{2}$-spaces, with the weight $\eta_{\beta}^{\alpha}$.

Furthermore, for $r=|\mathbf{x}|$ sufficiently large, we obtain the following anisotropic estimates:

$$
\begin{aligned}
& |\mathcal{O}(\mathbf{x})| \leq C r^{-1}(1+s)^{-2}, \quad\left|\frac{\partial \mathcal{O}}{\partial x_{1}}(\mathbf{x})\right| \leq C r^{-2}(1+s)^{-\frac{3}{2}}, \\
& \left|\frac{\partial \mathcal{O}}{\partial x_{j}}(\mathbf{x})\right| \leq C r^{-\frac{3}{2}}(1+s)^{-\frac{3}{2}}\left(1+\frac{2}{r}\right), \quad j=2,3, \quad \text { if } n=3, \\
& |\mathcal{O}(\mathbf{x})| \leq C r^{-\frac{1}{2}}(1+s)^{-1}, \quad\left|\frac{\partial \mathcal{O}}{\partial x_{1}}(\mathbf{x})\right| \leq C r^{-\frac{3}{2}}(1+s)^{-1}, \\
& \left|\frac{\partial \mathcal{O}}{\partial x_{2}}(\mathbf{x})\right| \leq C r^{-1}(1+s)^{-1}, \quad \text { if } n=2 .
\end{aligned}
$$

Note also the following properties:

$$
\begin{gathered}
\left.\forall p>3, \mathcal{O} \in L^{p}\left(\mathbb{R}^{3}\right) \quad \text { and } \quad \forall p \in\right] \frac{3}{2}, 2\left[, \quad \nabla \mathcal{O} \in \mathbf{L}^{p}\left(\mathbb{R}^{3}\right),\right. \\
\forall p \in] 2,3\left[, \quad \mathcal{O} \in L^{p}\left(\mathbb{R}^{3}\right) \quad \text { and } \quad \forall p \in\right] \frac{4}{3}, \frac{3}{2}\left[, \quad \nabla \mathcal{O} \in \mathbf{L}^{p}\left(\mathbb{R}^{3}\right),\right. \\
\mathcal{O} \in L_{l o c}^{1}\left(\mathbb{R}^{n}\right) \quad \text { and } \quad \nabla \mathcal{O} \in \mathbf{L}_{l o c}^{1}\left(\mathbb{R}^{n}\right), \quad \text { for } n=2,3 .
\end{gathered}
$$


Observe that when $\mathbf{f} \in \mathcal{D}\left(\mathbb{R}^{3}\right)$, then $u=O * f$ is a solution of (1.5). We have also $u=\mathcal{F}^{-1}\left(m_{0}(\xi) \mathcal{F} f\right)$, with $m_{0}(\xi)=\left(|\xi|^{2}+i k \xi_{1}\right)^{-1}$ and $\frac{\partial u}{\partial x_{j}}=\mathcal{F}^{-1}\left(m_{1}(\xi) \mathcal{F} f\right)$, with $m_{1}(\xi)=i \xi_{j}\left(|\xi|^{2}+i k \xi_{1}\right)^{-1}$. Here $\mathcal{F} f$ is the Fourier transform of $f$.

\section{Scalar Oseen Potential in three dimensional space}

This section is devoted to the $L^{p}$ estimates of convolutions with Oseen kernels. Before that, we introduce some basic weighted Sobolev spaces. We first set $\rho(\mathbf{x})=$ $\left(1+|\mathbf{x}|^{2}\right)^{\frac{1}{2}}, \lg \rho=\ln (1+\rho)$ and we define

$$
W_{0}^{1, p}\left(\mathbb{R}^{3}\right)=\left\{v \in \mathcal{D}^{\prime}\left(\mathbb{R}^{3}\right) ; \frac{v}{\omega_{1}} \in L^{p}, \nabla v \in L^{p}\left(\mathbb{R}^{3}\right)\right\},
$$

with $\omega_{1}=\rho$ if $p \neq 3, \omega_{1}=\rho \lg \rho$ if $p=3$ and $W_{0}^{-1, p^{\prime}}\left(\mathbb{R}^{3}\right)=\left(W_{0}^{1, p}\left(\mathbb{R}^{3}\right)\right)^{\prime}$. We recall that $\mathcal{D}\left(\mathbb{R}^{3}\right)$ is dense in $W_{0}^{1, p}\left(\mathbb{R}^{3}\right)$ and the constant functions belong to $W_{0}^{1, p}\left(\mathbb{R}^{3}\right)$ if $p \geq 3$. We now introduce a second family of weighted spaces:

$$
\widetilde{W}_{0}^{1, p}\left(\mathbb{R}^{n}\right)=\left\{v \in W_{0}^{1, p}\left(\mathbb{R}^{n}\right), \frac{\partial v}{\partial x_{1}} \in W_{0}^{-1, p}\left(\mathbb{R}^{n}\right)\right\}
$$

and we can prove that

$$
\mathcal{D}\left(\mathbb{R}^{n}\right) \text { is dense in } \widetilde{W}_{0}^{1, p}\left(\mathbb{R}^{n}\right) .
$$

Theorem 2.1. Let $f \in L^{p}\left(\mathbb{R}^{3}\right)$. Then $\frac{\partial^{2} \mathcal{O}}{\partial x_{j} \partial x_{k}} * f \in L^{p}\left(\mathbb{R}^{3}\right)$ (in the sense of principal value), $\frac{\partial \mathcal{O}}{\partial x_{1}} * f \in L^{p}\left(\mathbb{R}^{3}\right)$ and the following estimate holds

$$
\left\|\frac{\partial^{2} \mathcal{O}}{\partial x_{j} \partial x_{k}} * f\right\|_{L^{p}\left(\mathbb{R}^{3}\right)}+\left\|\frac{\partial \mathcal{O}}{\partial x_{1}} * f\right\|_{L^{p}\left(\mathbb{R}^{3}\right)} \leq C\|f\|_{L^{p}\left(\mathbb{R}^{3}\right)} .
$$

Moreover,

1) if $1<p<2$, then $\mathcal{O} * f \in L^{\frac{2 p}{2-p}}\left(\mathbb{R}^{3}\right)$ and satisfies

$$
\|\mathcal{O} * f\|_{L^{\frac{2 p}{2-p}\left(\mathbb{R}^{3}\right)}} \leq C\|f\|_{L^{p}\left(\mathbb{R}^{3}\right)} .
$$

2) If $1<p<4$, then $\frac{\partial \mathcal{O}}{\partial x_{j}} * f \in L^{\frac{4 p}{4-p}}\left(\mathbb{R}^{3}\right)$ and verifies the estimate

$$
\left\|\frac{\partial \mathcal{O}}{\partial x_{j}} * f\right\|_{L^{\frac{4 p}{4-p}\left(\mathbb{R}^{3}\right)}} \leq C\|f\|_{L^{p}\left(\mathbb{R}^{3}\right)} .
$$

Proof. By Fourier's transform, from Equation (1.5) we obtain:

$$
\mathcal{F}\left(\frac{\partial^{2} \mathcal{O}}{\partial x_{j} \partial x_{k}} * f\right)=\frac{-\xi_{j} \xi_{k}}{\xi^{2}+i \xi_{1}} \mathcal{F}(f) .
$$


Now, the function $\xi \mapsto m(\xi)=\frac{-\xi_{j} \xi_{k}}{\xi^{2}+i \xi_{1}}$ is of class $\mathcal{C}^{2}$ in $\mathbb{R}^{3} \backslash\{0\}$ and satisfies for every $\alpha=\left(\alpha_{1}, \alpha_{2}, \alpha_{3}\right) \in \mathbb{N}^{3}$

$$
\left|\frac{\partial^{|\alpha|} m}{\partial \boldsymbol{\xi}^{\alpha}}(\boldsymbol{\xi})\right| \leq C|\boldsymbol{\xi}|^{-\alpha},
$$

where, $|\alpha|=\alpha_{1}+\alpha_{2}+\alpha_{3}$ and $C$ is a constant not depending on $\boldsymbol{\xi}$. Then, the linear operator

$$
\mathcal{A}: f \mapsto \frac{\partial^{2} \mathcal{O}}{\partial x_{j} \partial x_{k}} * f(\mathbf{x})=\int_{\mathbb{R}^{3}} e^{i \mathbf{x} \boldsymbol{\xi}} \frac{-\xi_{j} \xi_{k}}{\boldsymbol{\xi}^{2}+i \xi_{1}} \mathcal{F} f(\boldsymbol{\xi}) d \boldsymbol{\xi}
$$

is continuous from $L^{p}\left(\mathbb{R}^{3}\right)$ into $L^{p}\left(\mathbb{R}^{3}\right)$ (see E. Stein [20], Thm 3.2, p.96). Therefore, $\frac{\partial^{2} \mathcal{O}}{\partial x_{j} \partial x_{k}} * f \in L^{p}\left(\mathbb{R}^{3}\right)$ and satisfies

$$
\left\|\frac{\partial^{2} \mathcal{O}}{\partial x_{j} \partial x_{k}} * f\right\|_{L^{p}\left(\mathbb{R}^{3}\right)} \leq C\|f\|_{L^{p}\left(\mathbb{R}^{3}\right)} .
$$

We also have

$$
\mathcal{F}\left(\frac{\partial \mathcal{O}}{\partial x_{1}} * f\right)=\frac{i \xi_{1}}{\boldsymbol{\xi}^{2}+i \xi_{1}} \mathcal{F}(f)
$$

and since the function $\boldsymbol{\xi} \mapsto m_{1}(\boldsymbol{\xi})=\frac{i \xi_{1}}{\overline{\boldsymbol{\xi}}^{2}+i \xi_{1}}$ have the same properties than $m(\boldsymbol{\xi})$, it follows that $\frac{\partial \mathcal{O}}{\partial x_{1}} * f \in L^{p}\left(\mathbb{R}^{3}\right)$ and satisfies the estimate

$$
\left\|\frac{\partial \mathcal{O}}{\partial x_{1}} * f\right\|_{L^{p}\left(\mathbb{R}^{3}\right)} \leq C\|f\|_{L^{p}\left(\mathbb{R}^{3}\right)},
$$

which proves the first part of the proposition and Estimate (2.1). Next, to prove inequalities (2.2) and (2.3), we adapt the technique used by Stein in [20] who studied the convolution of $f \in L^{p}\left(\mathbb{R}^{n}\right)$ with the kernel $|\mathbf{x}|^{\alpha-n}$. Let us decompose the function $K$ as $K_{1}+K_{\infty}$ where,

$$
\begin{aligned}
& K_{1}(\mathbf{x})=K(\mathbf{x}) \quad \text { if }|\mathbf{x}| \leq \mu \text { and } \quad K_{1}(\mathbf{x})=0 \quad \text { if }|\mathbf{x}|>\mu, \\
& K_{\infty}(\mathbf{x})=0 \quad \text { if }|\mathbf{x}| \leq \mu \text { and } K_{\infty}(\mathbf{x})=K(\mathbf{x}) \text { if }|\mathbf{x}|>\mu .
\end{aligned}
$$

The function $K$ will denote successively $\mathcal{O}$ and $\frac{\partial \mathcal{O}}{\partial x_{j}}$ and $\mu$ is a fixed positive constant which need not be specified at this instance. Next, we shall show that the mapping $f \mapsto K * f$ is of weak-type $(p, q)$, with $q=\frac{2 p}{2-p}$ when $K=\mathcal{O}$ and $q=\frac{4 p}{4-p}$ when $K=\frac{\partial \mathcal{O}}{\partial x_{j}}$, in the sense that:

$$
\text { for all } \lambda>0, \quad \operatorname{mes}\{\mathbf{x} ;|(K * f)(\mathbf{x})|>\lambda\} \leq\left(C_{p, q} \frac{\|f\|_{L^{p}\left(\mathbb{R}^{3}\right)}}{\lambda}\right)^{q} .
$$

Since $K * f=K_{1} * f+K_{\infty} * f$, we have now:

$$
\operatorname{mes}\{\mathbf{x} ;|K * f|>2 \lambda\} \leq \operatorname{mes}\left\{\mathbf{x} ;\left|K_{1} * f\right|>\lambda\right\}+\operatorname{mes}\left\{\mathbf{x} ;\left|K_{\infty} * f\right|>\lambda\right\} \text {. }
$$

Note that it is enough to prove inequality (2.5) with $\|f\|_{L^{p}\left(\mathbb{R}^{3}\right)}=1$. We have also:

$$
\operatorname{mes}\left\{\mathbf{x} ;\left|\left(K_{1} * f\right)(\mathbf{x})\right|>\lambda\right\} \leq \frac{\left\|K_{1} * f\right\|_{L^{p}\left(\mathbb{R}^{3}\right)}^{p}}{\lambda^{p}} \leq \frac{\left\|K_{1}\right\|_{L^{1}\left(\mathbb{R}^{3}\right)}^{p}}{\lambda^{p}},
$$


and

$$
\left\|K_{\infty} * f\right\|_{L^{\infty}\left(\mathbb{R}^{3}\right)} \leq\left\|K_{\infty}\right\|_{L^{p^{\prime}}\left(\mathbb{R}^{3}\right)} .
$$

1) Estimate (2.2). Observe that $\mathcal{O}_{1} \in L^{1}\left(\mathbb{R}^{3}\right)$ and $\mathcal{O}_{\infty} \in L^{p^{\prime}}\left(\mathbb{R}^{3}\right)$ for $1 \leq p<2$. Then, the integral $\mathcal{O}_{1} * f$ converges almost everywhere and $\mathcal{O}_{\infty} * f$ converges everywhere. Thus, $\mathcal{O} * f$ converges almost everywhere. But

$$
\forall \mu>0, \quad\left\|\mathcal{O}_{1}\right\|_{L^{1}\left(\mathbb{R}^{3}\right)} \leq C \mu .
$$

Next, by using (1.7), we have for any $p^{\prime}>2$ :

$$
\forall \mu>0, \quad\left\|\mathcal{O}_{\infty}\right\|_{L^{p^{\prime}}\left(\mathbb{R}^{3}\right)} \leq C \mu^{\frac{2-p^{\prime}}{p^{\prime}}} .
$$

Choosing now $\lambda=C \mu^{\frac{2-p^{\prime}}{p^{\prime}}}$ or equivalently $\mu=C^{\prime} \lambda^{\frac{p}{p-2}}$. Then from (2.10) and (2.8) we have $\left\|\mathcal{O}_{\infty} * f\right\|_{L^{\infty}\left(\mathbb{R}^{3}\right)}<\lambda$ and so mes $\left\{\mathbf{x} ;\left|\mathcal{O}_{\infty} * f\right|>\lambda\right\}=0$. Finally, for $1 \leq p<2$, we get from inequalities (2.9), (2.6) and (2.7):

$$
\operatorname{mes}\left\{\mathbf{x} \in \mathbb{R}^{3} ;|(\mathcal{O} * f)(\mathbf{x})|>\lambda\right\} \leq\left(C_{p} \frac{1}{\lambda}\right)^{\frac{2 p}{2-p}} .
$$

Therefore, for $1 \leq p<2$, the operator $R: f \mapsto \mathcal{O} * f$ is of weak-type $\left(p, \frac{2 p}{2-p}\right)$.

2) Estimate (2.3). Here we take $K=\frac{\partial \mathcal{O}}{\partial x_{j}}$. First, according to (2.1), $\frac{\partial \mathcal{O}}{\partial x_{1}} * f \in$ $W^{1, p}\left(\mathbb{R}^{3}\right)$ then, by the Sobolev embedding results, we have in particular, $\frac{\partial \mathcal{O}}{\partial x_{1}} * f \in$ $L^{\frac{4 p}{4-p}}\left(\mathbb{R}^{3}\right)$. It remains to prove Estimate $(2.3)$ for $j=2,3$. First we have:

$$
\left\|\frac{\partial \mathcal{O}}{\partial x_{j}}\right\|_{L^{1}\left(\mathbb{R}^{3}\right)} \leq c \mu \text {, if } \mu \leq 1 \text { and }\left\|\frac{\partial \mathcal{O}}{\partial x_{j}}\right\|_{L^{1}\left(\mathbb{R}^{3}\right)} \leq c \mu^{\frac{1}{2}} \text {, if } \mu>1 .
$$

Furthermore, we have for $p^{\prime}>\frac{4}{3}$ :

$$
\begin{aligned}
& \int_{|\mathbf{x}|>\mu}\left|\frac{\partial \mathcal{O}}{\partial x_{j}}(\mathbf{x})\right|^{p^{\prime}} d \mathbf{x} \leq C \mu^{4-3 p^{\prime}}, \quad \text { if } \mu \leq 1, \\
& \int_{|\mathbf{x}|>\mu}\left|\frac{\partial \mathcal{O}}{\partial x_{j}}(\mathbf{x})\right|^{p^{\prime}} d \mathbf{x} \leq C \mu^{\frac{4-3 p^{\prime}}{2}}, \quad \text { if } \mu>1 .
\end{aligned}
$$

Summarising we obtain:

a) If $0<\mu<1$

$$
\int_{|\mathbf{x}|<\mu}\left|\frac{\partial \mathcal{O}}{\partial x_{j}}(\mathbf{x})\right| d \mathbf{x} \leq c \mu \text { and } \int_{|\mathbf{x}|>\mu}\left|\frac{\partial \mathcal{O}}{\partial x_{j}}(\mathbf{x})\right|^{p^{\prime}} d \mathbf{x} \leq C \mu^{4-3 p^{\prime}},
$$

b) if $\mu \geq 1$,

$$
\int_{|\mathbf{x}|<\mu}\left|\frac{\partial \mathcal{O}}{\partial x_{j}}(\mathbf{x})\right| d \mathbf{x} \leq c \mu^{\frac{1}{2}} \quad \text { and } \quad \int_{|\mathbf{x}|>\mu}\left|\frac{\partial \mathcal{O}}{\partial x_{j}}(\mathbf{x})\right|^{p^{\prime}} d \mathbf{x} \leq C \mu^{\frac{4-3 p^{\prime}}{2}} .
$$


Setting $\lambda=C \mu^{\frac{4-3 p^{\prime}}{p^{\prime}}}$ in the case a) or $\lambda=C \mu^{\frac{4-3 p^{\prime}}{2 p^{\prime}}}$ in the case b), we get in both cases:

$$
\operatorname{mes}\left\{\mathbf{x} \in \mathbb{R}^{3} ;|K * f(\mathbf{x})|>\lambda\right\} \leq\left(C_{p} \frac{1}{\lambda}\right)^{\frac{4 p}{4-p}} .
$$

Thus, for $1 \leq p<4$, the operator $R_{j}: f \mapsto \frac{\partial \mathcal{O}}{\partial x_{j}} * f$ is of weak-type $\left(p, \frac{4 p}{4-p}\right)$. Applying now the Marcinkiewicz interpolation's theorem, we deduce that, for $1<$ $p<2$, the linear operator $R$ is continuous from $L^{p}\left(\mathbb{R}^{3}\right)$ into $L^{\frac{2 p}{2-p}}\left(\mathbb{R}^{3}\right)$ and for $1<p<4, R_{j}$ is continuous from $L^{p}\left(\mathbb{R}^{3}\right)$ into $L^{\frac{4 p}{4-p}}\left(\mathbb{R}^{3}\right)$.

Remark 2.2. Another proof of Theorem 2.1 consists in using Fourier's approach. Let $\left(f_{j}\right)_{j \in \mathbb{N}} \subset \mathcal{D}\left(\mathbb{R}^{3}\right)$ be a sequence which converges to $f \in L^{p}\left(\mathbb{R}^{3}\right)$. Then the sequence $\left(u_{j}\right)_{j \in \mathbb{N}}$ given by:

$$
u_{j}=\mathcal{F}^{-1}\left(m_{0}(\boldsymbol{\xi}) \mathcal{F} f_{j}\right), \quad m_{0}(\boldsymbol{\xi})=\left(|\boldsymbol{\xi}|^{2}+i \xi_{1}\right)^{-1},
$$

satisfies the equation $-\Delta u_{j}+\frac{\partial u_{j}}{\partial x_{1}}=f_{j}$. Let us recall now the:

Lizorkin Theorem. Let $D=\left\{\boldsymbol{\xi} \in \mathbb{R}^{3} ;|\boldsymbol{\xi}|>0\right\}$ and $m: D \longrightarrow \mathbb{C}$, a continuous function such that its derivatives $\frac{\partial^{k} m}{\partial \xi_{1}^{k_{1}} \partial \xi_{2}^{k_{2}} \partial \xi_{3}^{k_{3}}}$ are continuous and verify

$$
\left|\xi_{1}\right|^{k_{1}+\beta}\left|\xi_{2}\right|^{k_{2}+\beta}\left|\xi_{3}\right|^{k_{3}+\beta}\left|\frac{\partial^{k} m}{\partial \xi_{1}^{k_{1}} \partial \xi_{2}^{k_{2}} \partial \xi_{3}^{k_{3}}}\right| \leq M,
$$

where $k_{1}, k_{2}, k_{3} \in\{0,1\}, k=k_{1}+k_{2}+k_{3}$ and $0 \leq \beta<1$. Then, the operator

$$
\mathcal{A}: g \longmapsto \mathcal{F}^{-1}\left(m_{0} \mathcal{F} g\right),
$$

is continuous from $L^{p}\left(\mathbb{R}^{3}\right)$ into $L^{r}\left(\mathbb{R}^{3}\right)$ with $\frac{1}{r}=\frac{1}{p}-\beta$.

Applying this continuity property with $f_{j} \in L^{p}\left(\mathbb{R}^{3}\right)$ and $\beta=\frac{1}{2}$, we show that $\left(u_{j}\right)$ is bounded in $L^{\frac{2 p}{2-p}}\left(\mathbb{R}^{3}\right)$ if $1<p<2$. Thus, this sequence has a subsequence still denoted by $\left(u_{j}\right)$ which converges weakly to $u$ and which satisfies $T u=f$. For the derivative of $u_{j}$ with respect to $x_{1}$, the corresponding multiplier is of the form $m(\boldsymbol{\xi})=i \xi_{1}\left(|\boldsymbol{\xi}|^{2}+i \xi_{1}\right)^{-1}$. It follows that (2.14) is satisfied for $\beta=0$ and $\frac{\partial u}{\partial x_{1}} \in L^{p}\left(\mathbb{R}^{3}\right)$. The same property takes place for the derivatives of second order with $m(\boldsymbol{\xi})=\xi_{k} \xi_{l}\left(|\boldsymbol{\xi}|^{2}+i \xi_{1}\right)^{-1}$. Finally, we verify with $\beta=\frac{1}{4}$, that the derivative of $\left(u_{j}\right)$ with respect to $x_{k}$ is bounded in $L^{\frac{4 p}{4-p}}\left(\mathbb{R}^{3}\right)$, which implies $\frac{\partial u}{\partial x_{k}} \in L^{\frac{4 p}{4-p}}\left(\mathbb{R}^{3}\right)$.

Theorem 2.1 states that $\frac{\partial^{2} \mathcal{O}}{\partial x_{j} \partial x_{k}} * f \in L^{p}\left(\mathbb{R}^{3}\right)$ and under some conditions on $p$, $\frac{\partial \mathcal{O}}{\partial x_{j}} * f \in L^{\frac{4 p}{4-p}}\left(\mathbb{R}^{3}\right)$ and $\mathcal{O} * f \in L^{\frac{2 p}{2-p}}\left(\mathbb{R}^{3}\right)$. Now, using these results and the classical Sobolev embedding results, we have the following:

Theorem 2.3. Let $f \in L^{p}\left(\mathbb{R}^{3}\right)$.

1) Assume that $1<p<4$. Then $\nabla \mathcal{O} * f \in \mathbf{L}^{\frac{4 p}{4-p}}\left(\mathbb{R}^{3}\right)$ with the estimate (2.3). 
Moreover,

i) if $1<p<3$, then $\nabla \mathcal{O} * f \in \mathbf{L}^{\frac{3 p}{3-p}}\left(\mathbb{R}^{3}\right)$ with the estimate

$$
\|\nabla \mathcal{O} * f\|_{\mathbf{L}^{\frac{3 p}{3-p}}\left(\mathbb{R}^{3}\right)} \leq C\|f\|_{L^{p}\left(\mathbb{R}^{3}\right)} .
$$

ii) If $p=3$, then $\nabla \mathcal{O} * f \in \mathbf{L}^{r}\left(\mathbb{R}^{3}\right)$ for any $r \geq 12$ and satisfies

$$
\|\nabla \mathcal{O} * f\|_{\mathbf{L}^{r}\left(\mathbb{R}^{3}\right)} \leq C\|f\|_{L^{p}\left(\mathbb{R}^{3}\right)} .
$$

iii) If $3<p<4$, then $\nabla \mathcal{O} * f \in \mathbf{L}^{\infty}\left(\mathbb{R}^{3}\right)$ and verifies the estimate

$$
\|\nabla \mathcal{O} * f\|_{\mathbf{L}^{\infty}\left(\mathbb{R}^{3}\right)} \leq C\|f\|_{L^{p}\left(\mathbb{R}^{3}\right)} .
$$

2) Assume that $1<p<2$. Then $\mathcal{O} * f \in L^{\frac{2 p}{2-p}}\left(\mathbb{R}^{3}\right)$ with the estimate (2.2). Moreover,

i) if $1<p<\frac{3}{2}$, then $\mathcal{O} * f \in L^{\frac{3 p}{3-2 p}}\left(\mathbb{R}^{3}\right)$ and satisfies

$$
\|\mathcal{O} * f\|_{L^{\frac{3 p}{3-2 p}\left(\mathbb{R}^{3}\right)}} \leq C\|f\|_{L^{p}\left(\mathbb{R}^{3}\right)} .
$$

ii) If $p=\frac{3}{2}$, then $\mathcal{O} * f \in L^{r}\left(\mathbb{R}^{3}\right)$ for any $r \geq 6$ and

$$
\|\mathcal{O} * f\|_{L^{r}\left(\mathbb{R}^{3}\right)} \leq C\|f\|_{L^{p}\left(\mathbb{R}^{3}\right)} .
$$

iii) If $\frac{3}{2}<p<2$, then $\mathcal{O} * f \in L^{\infty}\left(\mathbb{R}^{3}\right)$ and the following estimate holds

$$
\|\mathcal{O} * f\|_{L^{\infty}\left(\mathbb{R}^{3}\right)} \leq C\|f\|_{L^{p}\left(\mathbb{R}^{3}\right)} .
$$

Proof. 1) If $1<p<4$, the previous theorem asserts that $\frac{\partial \mathcal{O}}{\partial x_{j}} * f \in L^{\frac{4 p}{4-p}}\left(\mathbb{R}^{3}\right)$ and $\frac{\partial^{2} \mathcal{O}}{\partial x_{j} \partial x_{k}} * f \in L^{p}\left(\mathbb{R}^{3}\right)$. If $1<p<3$, there exists a unique constant $k(f) \in \mathbb{R}$ such that $v=\frac{\partial \mathcal{O}}{\partial x_{j}} * f+k(f) \in W_{0}^{1, p}\left(\mathbb{R}^{3}\right)$. Then $k(f)=v-\frac{\partial \mathcal{O}}{\partial x_{j}} * f \in W_{0}^{1, p}\left(\mathbb{R}^{3}\right)+L^{\frac{4 p}{4-p}}\left(\mathbb{R}^{3}\right)$. As none of both spaces contains constants then $k(f)=0$, which implies that $\frac{\partial \mathcal{O}}{\partial x_{j}} * f \in W_{0}^{1, p}\left(\mathbb{R}^{3}\right)$. Now, the Sobolev embedding results yield $\frac{\partial \mathcal{O}}{\partial x_{j}} * f \in L^{\frac{3 p}{3-p}}\left(\mathbb{R}^{3}\right)$ and Estimate (2.15). If $p \geq 3$, again by the previous theorem, we have $\frac{\partial \mathcal{O}}{\partial x_{j}} * f \in$ $W_{0}^{1, p}\left(\mathbb{R}^{3}\right)$. Then $\frac{\partial \mathcal{O}}{\partial x_{j}} * f \in B M O\left(\mathbb{R}^{3}\right)$ if $p=3$. Applying now the interpolation theorem between $B M O\left(\mathbb{R}^{3}\right)$ and $L^{p}\left(\mathbb{R}^{3}\right)$, we get $\frac{\partial \mathcal{O}}{\partial x_{j}} * f \in L^{r}\left(\mathbb{R}^{3}\right)$ for any $r \geq 12$. By Sobolev embedding results, if $3<p<4$, we have $\frac{\partial \mathcal{O}}{\partial x_{j}} * f \in L^{\infty}\left(\mathbb{R}^{3}\right)$, ) and the case 1) is proved.

2) By the previous theorem, if $1<p<2$, we have $\mathcal{O} * f \in L^{\frac{2 p}{2-p}}\left(\mathbb{R}^{3}\right)$ and $\nabla \mathcal{O} * f \in$ $\mathbf{L}^{\frac{3 p}{3-p}}\left(\mathbb{R}^{3}\right)$. Now by Sobolev embedding results, $\mathcal{O} * f \in L^{p^{*}}\left(\mathbb{R}^{3}\right)$, where $\frac{1}{p^{*}}=$ $\frac{3-p}{3 p}-\frac{1}{3}=\frac{1}{p}-\frac{2}{3}$ if $1<p<\frac{3}{2}$, which yields (2.15). For the remainder of the proof, we use the same arguments that in the previous case with $\mathcal{O} * f$ instead of $\frac{\partial \mathcal{O}}{\partial x_{j}} * f$ and $\frac{\partial \mathcal{O}}{\partial x_{j}} * f$ instead of $\frac{\partial^{2} \mathcal{O}}{\partial x_{j} \partial x_{k}} * f$.

Remark 2.4. In Farwig and Sohr [8], Theorem 2.3 proves existence of solutions to the Oseen equations with forces in $L^{p}$, thanks to the Lizorkin theorem's. These solutions, which are not explicit, belong to homogeneous Sobolev spaces. Here, in 
Theorem 2.1, we prove some continuity properties for the Oseen potential, without using Lizorkin theorem's, and in Theorem 2.3, we complete thoses properties, thanks to Sobolev embeddings and we find the same results as the ones given in [8].

Remark 2.5. i) We can also have the result given by Theorem 2.32 ), by showing that $\mathcal{O} \in L^{2, \infty}\left(\mathbb{R}^{3}\right)$, i.e.

$$
\sup _{\mu>0} \mu^{2} \operatorname{mes}\left\{\mathbf{x} \in \mathbb{R}^{3} ; \mathcal{O}(\mathbf{x})>\mu\right\}<+\infty .
$$

So that, for any $1<q<2$, according to weak Young inequality ( $c f$. [19], chap. IX.4), we obtain:

$$
\|\mathcal{O} * f\|_{L^{\frac{2 q}{2-q}, \infty}\left(\mathbb{R}^{3}\right)} \leq C\|\mathcal{O}\|_{L^{2, \infty}\left(\mathbb{R}^{3}\right)}\|f\|_{L^{q}\left(\mathbb{R}^{3}\right)} .
$$

Let now $p \in] 1,2\left[\right.$. There exist $p_{0}$ and $p_{1}$ such that $1<p_{0}<p<p_{1}<2$ and such that the operator $R: f \longmapsto \mathcal{O} * f$ is continuous from $L^{p_{0}}\left(\mathbb{R}^{3}\right)$ into $L^{\frac{2 p_{0}}{2-p_{0}}, \infty}\left(\mathbb{R}^{3}\right)$ and from $L^{p_{1}}\left(\mathbb{R}^{3}\right)$ into $L^{\frac{2 p_{1}}{2-p_{1}}, \infty}\left(\mathbb{R}^{3}\right)$. The Marcinkiewicz theorem allows again to conclude that the operator $R$ is continuous from $L^{p}\left(\mathbb{R}^{3}\right)$ into $L^{\frac{2 p}{2-p}}\left(\mathbb{R}^{3}\right)$

ii) The same remark remains valid for $\nabla \mathcal{O}$ that belongs to $\mathbf{L}^{\frac{4}{3}, \infty}\left(\mathbb{R}^{3}\right)$.

Using the Young inequality with the relations (1.10) and (1.11), we get the following result:

Proposition 2.6. Let $f \in L^{1}\left(\mathbb{R}^{3}\right)$. Then

1) $\mathcal{O} * f \in L^{p}\left(\mathbb{R}^{3}\right)$ for any $\left.p \in\right] 2,3[$ and satisfies the estimate

$$
\|\mathcal{O} * f\|_{L^{p}\left(\mathbb{R}^{3}\right)} \leq C\|f\|_{L^{1}\left(\mathbb{R}^{3}\right)},
$$

2) $\nabla \mathcal{O} * f \in \mathbf{L}^{p}\left(\mathbb{R}^{3}\right)$ for any $\left.p \in\right] \frac{4}{3}, \frac{3}{2}$ [ and the following estimate holds

$$
\|\nabla \mathcal{O} * f\|_{\mathbf{L}^{p}\left(\mathbb{R}^{3}\right)} \leq C\|f\|_{L^{1}\left(\mathbb{R}^{3}\right)} .
$$

Remark 2.7. Taking "formally" $p=1$ in Theorem 2.3 , we find that $\mathcal{O} * f \in L^{q}\left(\mathbb{R}^{3}\right)$ for any $q \in] 2,3\left[\right.$ and $\nabla \mathcal{O} * f \in L^{q}\left(\mathbb{R}^{3}\right)$ for any $\left.q \in\right] \frac{4}{3}, \frac{3}{2}[$. We notice that they are the same results obtained in Theorem 2.6 by using the Young inequality.

Now, we are going to study the Oseen potential $\mathcal{O} * f$ when $f$ belongs to $W_{0}^{-1, p}\left(\mathbb{R}^{3}\right)$. For that purpose, we give the following definition of the convolution of $f$ with the fundamental solution $\mathcal{O}$ :

$$
\forall \varphi \in \mathcal{D}\left(\mathbb{R}^{3}\right), \quad\langle\mathcal{O} * f, \varphi\rangle=:\langle f, \breve{\mathcal{O}} * \varphi\rangle_{W_{0}^{-1, p}\left(\mathbb{R}^{3}\right) \times W_{0}^{1, p^{\prime}}\left(\mathbb{R}^{3}\right)},
$$

where $\breve{\mathcal{O}}(\mathbf{x})=\mathcal{O}(-\mathbf{x})$. With the $L^{\infty}$ weighted estimates obtained in [14] (Thms 3.1 and 3.2$)$, we get an estimate on the convolution of $\breve{\mathcal{O}}$ with a function $\varphi \in \mathcal{D}\left(\mathbb{R}^{3}\right)$ which we shall use afterward as follow

Lemma 2.8. For any $\varphi \in \mathcal{D}\left(\mathbb{R}^{3}\right)$ we have the estimates

$$
|\breve{\mathcal{O}} * \varphi(\mathbf{x})| \leq C_{\varphi} \frac{1}{|\mathbf{x}|\left(1+|\mathbf{x}|+x_{1}\right)},
$$




$$
|\nabla \breve{\mathcal{O}} * \varphi(\mathbf{x})| \leq C_{\varphi} \frac{1}{|\mathbf{x}|^{\frac{3}{2}}\left(1+|\mathbf{x}|+x_{1}\right)^{\frac{3}{2}}},
$$

where $C_{\varphi}$ depends on the support of $\varphi$.

Remark 2.9. 1) The behaviour on $|\mathbf{x}|$ of $\breve{\mathcal{O}} * \varphi$ and its first derivatives is the same that of $\breve{\mathcal{O}}$, but the behaviour on $1+s^{\prime}$ is slightly different (see (1.7).

2) From estimates $(2.26),(2.27)$ we find that

$$
\forall q>\frac{4}{3}, \quad \breve{\mathcal{O}} * \varphi \in W_{0}^{1, q}\left(\mathbb{R}^{3}\right) .
$$

3) In (2.26) and (2.27), when $\varphi$ tends to zero in $\mathcal{D}\left(\mathbb{R}^{3}\right)$, then $C_{\varphi}$ tends to zero in $\mathbb{R}$.

The next theorem studies the continuity of the operators $R$ and $R_{j}$ when $f$ belongs to $W_{0}^{-1, p}\left(\mathbb{R}^{3}\right)$.

Theorem 2.10. Assume that $1<p<4$ and let $f \in W_{0}^{-1, p}\left(\mathbb{R}^{3}\right)$ satisfying the compatibility condition

$$
\langle f, 1\rangle_{W_{0}^{-1, p}\left(\mathbb{R}^{3}\right) \times W_{0}^{1, p^{\prime}}\left(\mathbb{R}^{3}\right)}=0, \quad \text { when } 1<p \leq \frac{3}{2} .
$$

Then $\mathcal{O} * f \in L^{\frac{4 p}{4-p}}\left(\mathbb{R}^{3}\right)$ and $\nabla \mathcal{O} * f \in \mathbf{L}^{p}\left(\mathbb{R}^{3}\right)$ with the following estimate

$$
\|\mathcal{O} * f\|_{L^{\frac{4 p}{4-p}\left(\mathbb{R}^{3}\right)}}+\|\nabla \mathcal{O} * f\|_{\mathbf{L}^{p}\left(\mathbb{R}^{3}\right)} \leq C\|f\|_{W_{0}^{-1, p}\left(\mathbb{R}^{3}\right)} .
$$

Moreover,

i) if $1<p<3$, then $\mathcal{O} * f \in L^{\frac{3 p}{3-p}}\left(\mathbb{R}^{3}\right)$ and the following estimate holds

$$
\|\mathcal{O} * f\|_{L^{\frac{3 p}{3-p}}\left(\mathbb{R}^{3}\right)} \leq C\|f\|_{W_{0}^{-1, p}\left(\mathbb{R}^{3}\right)} .
$$

ii) If $p=3$, then $\mathcal{O} * f \in L^{r}\left(\mathbb{R}^{3}\right)$ for any $r \geq 12$ and satisfies

$$
\|\mathcal{O} * f\|_{L^{r}\left(\mathbb{R}^{3}\right)} \leq C\|f\|_{W_{0}^{-1, p}\left(\mathbb{R}^{3}\right)} .
$$

iii) If $3<p<4$, then $\mathcal{O} * f \in L^{\infty}\left(\mathbb{R}^{3}\right)$ and we have the estimate

$$
\|\mathcal{O} * f\|_{L^{\infty}\left(\mathbb{R}^{3}\right)} \leq C\|f\|_{W_{0}^{-1, p}\left(\mathbb{R}^{3}\right)} .
$$

Proof. Let $1<p<4$. By Lemma 2.8 and Remark 2.9 point 3), if $\varphi \rightarrow 0$ in $\mathcal{D}\left(\mathbb{R}^{3}\right)$, then $C_{\varphi} \rightarrow 0$ where $C_{\varphi}$ is defined by $(2.26)$. Thus, $\breve{\mathcal{O}} * \varphi \rightarrow 0$ in $W_{0}^{1, p^{\prime}}\left(\mathbb{R}^{3}\right)$ for all $p \in] 1,4\left[\right.$, which implies that $\mathcal{O} * f \in \mathcal{D}^{\prime}\left(\mathbb{R}^{3}\right)$. Next, there exists $\mathbf{F} \in \mathbf{L}^{p}\left(\mathbb{R}^{3}\right)$ such that

$$
f=\operatorname{div} \mathbf{F} \quad \text { and } \quad\|\mathbf{F}\|_{\mathbf{L}^{p}\left(\mathbb{R}^{3}\right)} \leq C\|f\|_{W_{0}^{-1, p}\left(\mathbb{R}^{3}\right)} .
$$

According to $(2.1)$, we have for any $\varphi \in \mathcal{D}\left(\mathbb{R}^{3}\right)$,

$$
\begin{aligned}
\left|\left\langle\frac{\partial \mathcal{O}}{\partial x_{j}} * f, \varphi\right\rangle_{\mathcal{D}^{\prime}\left(\mathbb{R}^{3}\right) \times \mathcal{D}\left(\mathbb{R}^{3}\right)}\right| & =\left|\left\langle\mathbf{F}, \nabla \frac{\partial}{\partial x_{j}} \breve{\mathcal{O}} * \varphi\right\rangle_{\mathbf{L}^{p}\left(\mathbb{R}^{3}\right) \times \mathbf{L}^{p^{\prime}}\left(\mathbb{R}^{3}\right)}\right| \\
& \leq C\|f\|_{W_{0}^{-1, p}\left(\mathbb{R}^{3}\right)}\|\varphi\|_{L^{p^{\prime}\left(\mathbb{R}^{3}\right)}} .
\end{aligned}
$$


Then we deduce the second part of (2.30). We also have for all $\varphi \in \mathcal{D}\left(\mathbb{R}^{3}\right)$ :

$$
\langle\mathcal{O} * f, \varphi\rangle_{\mathcal{D}^{\prime}\left(\mathbb{R}^{3}\right) \times \mathcal{D}\left(\mathbb{R}^{3}\right)}=-\langle\mathbf{F}, \nabla \breve{\mathcal{O}} * \varphi\rangle_{\mathbf{L}^{p}\left(\mathbb{R}^{3}\right) \times \mathbf{L}^{p^{\prime}}\left(\mathbb{R}^{3}\right)},
$$

and by (2.3):

$$
\left|\langle\mathcal{O} * f, \varphi\rangle_{\mathcal{D}^{\prime}\left(\mathbb{R}^{3}\right) \times \mathcal{D}\left(\mathbb{R}^{3}\right)}\right| \leq C\|f\|_{W_{0}^{-1, p}\left(\mathbb{R}^{3}\right)}\|\varphi\|_{L^{\frac{4 p}{5 p-4}\left(\mathbb{R}^{3}\right)}} .
$$

Note that $1<p<4 \Longleftrightarrow 1<\frac{4 p}{5 p-4}<4$. Consequently, we have the first part of (2.30). Moreover, by Sobolev embeddings, $\mathcal{O} * f \in L^{\frac{3 p}{3-p}}\left(\mathbb{R}^{3}\right)$ if $1<p<3, \mathcal{O} * f$ belongs to $L^{r}\left(\mathbb{R}^{3}\right)$ for all $r \geq 12$ if $p=3$ and belongs to $L^{\infty}\left(\mathbb{R}^{3}\right)$ if $3<p<4$. Thus, we showed that if $1<p<4$, the operators $R$ and $R_{j}$ are continuous.

Corollary 2.11. Assume that $1<p<4$. If $u$ is a distribution such that $\nabla u \in$ $\mathbf{L}^{p}\left(\mathbb{R}^{3}\right)$ and $\frac{\partial u}{\partial x_{1}} \in W_{0}^{-1, p}\left(\mathbb{R}^{3}\right)$, then there exists a unique constant $k(u)$ such that $u+k(u) \in L^{\frac{4 p}{4-p}}\left(\mathbb{R}^{3}\right)$ and

$$
\|u+k(u)\|_{L^{\frac{4 p}{4-p}}\left(\mathbb{R}^{3}\right)} \leq C\left(\|\nabla u\|_{\mathbf{L}^{p}\left(\mathbb{R}^{3}\right)}+\left\|\frac{\partial u}{\partial x_{1}}\right\|_{W_{0}^{-1, p}\left(\mathbb{R}^{3}\right)}\right) .
$$

Moreover, if $1<p<3$, then $u+k(u) \in L^{\frac{3 p}{3-p}}\left(\mathbb{R}^{3}\right)$, where $k(u)$ is defined by:

$$
k(u)=-\lim _{|\mathbf{x}| \rightarrow \infty} \frac{1}{\omega_{3}} \int_{S_{2}} u(\sigma|\mathbf{x}|) d \sigma,
$$

where, $\omega_{3}$ denotes the area of the sphere $S_{2}$ and $u$ tends to the constant $-k(u)$ as $\mathbf{x}$ tends to infinity in the following sense:

$$
\lim _{|\mathbf{x}| \rightarrow \infty} \int_{S_{2}}|u(\sigma|\mathbf{x}|)+k(u)| d \sigma=0 .
$$

If $p=3$, then $u+k(u)$ belongs to $L^{r}\left(\mathbb{R}^{3}\right)$ for any $r \geq 12$. If $3<p<4$, then $u$ belongs to $L^{\infty}\left(\mathbb{R}^{3}\right)$, is continuous in $\mathbb{R}^{3}$ and tends to $-k(u)$ pointwise.

Proof. We set $g=-\Delta u+\frac{\partial u}{\partial x_{1}} \in W_{0}^{-1, p}\left(\mathbb{R}^{3}\right)$. Since $\mathcal{P}_{\left[1-\frac{3}{p^{\prime}}\right]}$ contains at most constants and according to the density of $\mathcal{D}\left(\mathbb{R}^{3}\right)$ in $\widetilde{W}_{0}^{1, p}\left(\mathbb{R}^{3}\right)$, then $g$ satisfies the compatibility Condition (2.29). By the previous theorem, there exists a unique $v=\mathcal{O} * g \in L^{\frac{4 p}{4-p}}\left(\mathbb{R}^{3}\right)$ such that $\nabla v \in L^{p}\left(\mathbb{R}^{3}\right)$ and $\frac{\partial v}{\partial x_{1}} \in L^{p}\left(\mathbb{R}^{3}\right)$, satisfying $T(u-v)=0$, where $T$ is the Oseen operator, with the estimate:

$$
\|v\|_{L^{\frac{4 p}{4-p}}\left(\mathbb{R}^{3}\right)} \leq C\left(\|\nabla u\|_{\mathbf{L}^{p}\left(\mathbb{R}^{3}\right)}+\left\|\frac{\partial u}{\partial x_{1}}\right\|_{W_{0}^{-1, p}\left(\mathbb{R}^{3}\right)}\right) .
$$

Setting $w=u-v$, we have for all $i=1,2,3, \frac{\partial w}{\partial x_{i}} \in L^{p}\left(\mathbb{R}^{3}\right)$ and satisfies $T\left(\frac{\partial w}{\partial x_{i}}\right)=0$. Then by an uniqueness argument, we deduce that $\nabla u=\nabla v$ and consequently there exists a unique constant $k(u)$, defined by $(2.36)$, such that $u+k(u)=v$. The last properties are consequences of Sobolev embeddings. 
Remark 2.12. Let $u \in \mathcal{D}^{\prime}\left(\mathbb{R}^{3}\right)$ such that $\nabla u \in \mathbf{L}^{p}\left(\mathbb{R}^{3}\right)$.

i) If $1<p<3$, we know that there exists a unique constant $k(u)$ such that $u+k(u) \in L^{\frac{3 p}{3-p}}\left(\mathbb{R}^{3}\right)$. Here, the fact that in addition $\frac{\partial u}{\partial x_{1}} \in W_{0}^{-1, p}\left(\mathbb{R}^{3}\right)$ we also have $u+k(u) \in L^{\frac{4 p}{4-p}}\left(\mathbb{R}^{3}\right)$, with $\frac{4 p}{4-p}<\frac{3 p}{3-p}$.

ii) If $3 \leq p<4$, for any constant $k, u+k$ belongs only to $W_{0}^{1, p}\left(\mathbb{R}^{3}\right)$ but not to the space $L^{r}\left(\mathbb{R}^{3}\right)$. But, if moreover $\frac{\partial u}{\partial x_{1}} \in W_{0}^{-1, p}\left(\mathbb{R}^{3}\right)$ then, $u+k(u) \in L^{\frac{4 p}{4-p}}\left(\mathbb{R}^{3}\right)$ for some unique constant $k(u)$. Moreover $u+k(u) \in L^{r}\left(\mathbb{R}^{3}\right)$ for any $r \geq \frac{4 p}{4-p}$ and $u \in L^{\infty}\left(\mathbb{R}^{3}\right)$ if $p>3$.

\section{Weighted Hardy inequalities}

In this section, our aim is to give some weighted anisotropic Hardy inequalities in $\mathbb{R}^{n}$ with $n \geq 2$.

For $\alpha, \beta \in \mathbb{R}$, we consider the anisotropic weight functions

$$
\eta_{\beta}^{\alpha}=(1+r)^{\alpha}(1+s)^{\beta},
$$

with

$$
s=s(\mathbf{x})=r-x_{1} .
$$

We define the weighted space

$$
L_{\alpha, \beta}^{p}\left(\mathbb{R}^{n}\right)=\left\{v \in \mathcal{D}^{\prime}\left(\mathbb{R}^{n}\right), \quad \eta_{\beta}^{\alpha} v \in L^{p}\left(\mathbb{R}^{n}\right)\right\},
$$

which is a Banach space for its natural norm given by

$$
\|v\|_{L_{\alpha, \beta}^{p}\left(\mathbb{R}^{n}\right)}=\left\|\eta_{\beta}^{\alpha} v\right\|_{L^{p}\left(\mathbb{R}^{n}\right)} .
$$

We introduce the first family of weighted Sobolev spaces,

$$
\begin{aligned}
& W_{\alpha, \beta}^{1, p}\left(\mathbb{R}^{3}\right)=\left\{v \in L_{\alpha-\frac{1}{2}, \beta}^{p}\left(\mathbb{R}^{n}\right), \nabla v \in \mathbf{L}_{\alpha, \beta}^{p}\left(\mathbb{R}^{n}\right)\right\}, \\
& X_{\alpha, \beta}^{1, p}\left(\mathbb{R}^{3}\right)=\left\{v \in L_{\alpha-\frac{1}{2}, \beta-\frac{1}{2}}^{p}\left(\mathbb{R}^{n}\right), \nabla v \in \mathbf{L}_{\alpha, \beta}^{p}\left(\mathbb{R}^{n}\right)\right\}, \\
& Y_{\alpha, \beta}^{1, p}\left(\mathbb{R}^{3}\right)=\left\{v \in L_{\alpha-1, \beta}^{p}\left(\mathbb{R}^{n}\right), \nabla v \in \mathbf{L}_{\alpha, \beta}^{p}\left(\mathbb{R}^{n}\right)\right\} .
\end{aligned}
$$

These are Banach spaces for their natural norms. Observe that

$$
W_{\alpha, \beta}^{1, p}\left(\mathbb{R}^{3}\right) \subset X_{\alpha, \beta}^{1, p}\left(\mathbb{R}^{3}\right) \subset Y_{\alpha, \beta}^{1, p}\left(\mathbb{R}^{3}\right) .
$$

All the local properties of the spaces $W_{\alpha, \beta}^{1, p}\left(\mathbb{R}^{3}\right), X_{\alpha, \beta}^{1, p}\left(\mathbb{R}^{3}\right)$ and $Y_{\alpha, \beta}^{1, p}\left(\mathbb{R}^{3}\right)$ coincide with those of classical Sobolev spaces $W^{1, p}\left(\mathbb{R}^{n}\right)$. Moreover, we have the following properties:

\section{Proposition 3.1.}

The space $\mathcal{D}\left(\mathbb{R}^{n}\right)$ is dense in $W_{\alpha, \beta}^{1, p}\left(\mathbb{R}^{3}\right)\left(\right.$ resp. in $X_{\alpha, \beta}^{1, p}\left(\mathbb{R}^{3}\right)$ and in $\left.Y_{\alpha, \beta}^{1, p}\left(\mathbb{R}^{3}\right)\right)$. 
Proof. It relies on a truncation procedure. Let $u \in W_{\alpha, \beta}^{1, p}\left(\mathbb{R}^{3}\right), \varphi \in \mathcal{D}\left(\mathbb{R}^{n}\right)$, with $0 \leq \varphi(\mathbf{x}) \leq 1, \varphi(\mathbf{x})=1$ if $r \leq 1, \varphi(\mathbf{x})=0$ if $r \geq 2$, and set $\varphi_{k}(\mathbf{x})=\varphi(\mathbf{x} / k)$, $u_{k}=u \varphi_{k}$. We have

$$
\begin{aligned}
\left\|u_{k}-u\right\|_{W_{\alpha, \beta}^{1, p}\left(\mathbb{R}^{3}\right)}^{p} & =\left\|u_{k}-u\right\|_{L_{\alpha-\frac{1}{2}, \beta}^{p}\left(\mathbb{R}^{n}\right)}^{p}+\left\|\nabla\left(u_{k}-u\right)\right\|_{\mathbf{L}_{\alpha, \beta}^{p}\left(\mathbb{R}^{n}\right)}^{p} \\
& \leq\left\|\left(\varphi_{k}-1\right) u\right\|_{L_{\alpha-\frac{1}{2}, \beta}^{p}}^{p}\left(\mathbb{R}^{n}\right) \\
& +C\left\|\left(\varphi_{k}-1\right) \nabla u\right\|_{\mathbf{L}_{\alpha, \beta}^{p}\left(\mathbb{R}^{n}\right)}^{p} \\
& +C u \nabla \varphi_{k} \|_{\mathbf{L}_{\alpha, \beta}^{p}\left(\mathbb{R}^{n}\right)}^{p}
\end{aligned}
$$

where $C$ is a positive real. Since $u \in W_{\alpha, \beta}^{1, p}\left(\mathbb{R}^{3}\right)$, it is clear that the first two terms of the right hand side of (3.1) tend to zero, when $k$ tends to $\infty$. Now, the last term of (3.1) can be written,

$$
\left\|u \nabla \varphi_{k}\right\|_{\mathbf{L}_{\alpha, \beta}^{p}\left(\mathbb{R}^{n}\right)}^{p}=\int_{\{k \leq r \leq 2 k\}} \eta_{\beta p}^{\alpha p}\left|u \nabla \varphi_{k}\right|^{p} d \mathbf{x}
$$

and, since $\left|\nabla \varphi_{k}(\mathbf{x})\right| \leq \frac{1}{k}|\nabla \varphi(\mathbf{x} / k)|$, we arrive at

$$
\left\|u \nabla \varphi_{k}\right\|_{\mathbf{L}_{\alpha, \beta}^{p}\left(\mathbb{R}^{n}\right)}^{p} \leq C \int_{\{k \leq r \leq 2 k\}} \eta_{\beta p}^{(\alpha-1) p}|u|^{p} d \mathbf{x} .
$$

Recalling that $u \in W_{\alpha, \beta}^{1, p}\left(\mathbb{R}^{3}\right)$, this last quantity tends to zero as $k$ tends to $\infty$. Then, since each $u_{k}$ has a compact support and the topologies of $W_{\alpha, \beta}^{1, p}\left(\mathbb{R}^{3}\right)$ and $W^{1, p}\left(\mathbb{R}^{n}\right)$ coincide on this support, the statement of the proposition follows from the density of $\mathcal{D}\left(\mathbb{R}^{n}\right)$ in $W^{1, p}\left(\mathbb{R}^{n}\right)$. The proof is the same for the two other spaces.

The previous proposition implies that the dual spaces respectively denoted $W_{-\alpha,-\beta}^{-1, p^{\prime}}\left(\mathbb{R}^{n}\right)$, $X_{-\alpha,-\beta}^{-1, p^{\prime}}\left(\mathbb{R}^{n}\right), Y_{-\alpha,-\beta}^{-1, p^{\prime}}\left(\mathbb{R}^{n}\right)$ are subspaces of $\mathcal{D}^{\prime}\left(\mathbb{R}^{n}\right)$. Let $\rho$ be the weight function $\rho=1+r=\eta_{0}^{1}$ and $\lg r=\ln (1+\rho)$. For $\alpha \in \mathbb{R}$, we recall the following weighted Sobolev spaces

$$
\begin{aligned}
& W_{\alpha}^{0, p}\left(\mathbb{R}^{n}\right)=\left\{u \in \mathcal{D}^{\prime}\left(\mathbb{R}^{n}\right), \rho^{\alpha} u \in L^{p}\left(\mathbb{R}^{n}\right)\right\}=L_{\alpha, 0}^{p}\left(\mathbb{R}^{n}\right), \\
& W_{\alpha}^{1, p}\left(\mathbb{R}^{n}\right)=\left\{u \in W_{\alpha-1}^{0, p}\left(\mathbb{R}^{n}\right), \nabla u \in \mathbf{W}_{\alpha}^{0, p}\left(\mathbb{R}^{n}\right)\right\}, \text { if } \frac{n}{p}+\alpha \neq 1, \\
& W_{\alpha}^{1, p}\left(\mathbb{R}^{n}\right)=\left\{(\lg r)^{-1} u \in W_{\alpha-1}^{0, p}\left(\mathbb{R}^{n}\right), \nabla u \in \mathbf{W}_{\alpha}^{0, p}\left(\mathbb{R}^{n}\right)\right\}, \text { if } \frac{n}{p}+\alpha=1 .
\end{aligned}
$$

We have the following identity:

$$
W_{\alpha}^{1, p}\left(\mathbb{R}^{n}\right)=Y_{\alpha, 0}^{1, p}\left(\mathbb{R}^{n}\right) \quad \text { if } \frac{n}{p}+\alpha \neq 1
$$

We will now prove some one-dimensional inequalities. 
Lemma 3.2. Let $\gamma \in \mathbb{R}$ satisfy $\gamma+\frac{n-1}{2}>0$ and $\left.\theta^{*} \in\right] 0, \pi / 2[$. Then for any positive measurable function $f$ defined on $] 0, \theta^{*}[$, such that

$$
\int_{0}^{\theta^{*}}(1-\cos \theta)^{\gamma+\frac{p}{2}}(\sin \theta)^{n-2}[f(\theta)]^{p} d \theta<+\infty
$$

one has

$$
\int_{0}^{\theta^{*}}(1-\cos \theta)^{\gamma}(\sin \theta)^{n-2}[F(\theta)]^{p} d \theta \leq C \int_{0}^{\theta^{*}}(1-\cos \theta)^{\gamma+\frac{p}{2}}(\sin \theta)^{n-2}[f(\theta)]^{p} d \theta,
$$

with

$$
F(\theta)=\int_{\theta}^{\theta^{*}} f(t) d t
$$

Proof. Let us first notice that on $]-\frac{\pi}{2}, \frac{\pi}{2}[$, the following inequality holds

$$
\frac{1}{2} \sin ^{2} \theta \leq 1-\cos \theta \leq \sin ^{2} \theta \text {. }
$$

We now set

$$
J=\int_{0}^{\theta^{*}}(1-\cos \theta)^{\gamma}(\sin \theta)^{n-2}(F(\theta))^{p} d \theta
$$

In view of Inequality (3.7), we find

$$
\begin{aligned}
J & =\int_{0}^{\theta^{*}}(1-\cos \theta)^{\gamma}(\sin \theta)^{n-3} \sin \theta(F(\theta))^{p} d \theta \\
& \leq 2^{(n-3) / 2} \int_{0}^{\theta^{*}}(1-\cos \theta)^{\gamma+\frac{n-3}{2}} \sin \theta(F(\theta))^{p} d \theta .
\end{aligned}
$$

From (3.6) and since $\gamma+\frac{n-1}{2}>0$, an integration by parts yields

$$
J \leq C \int_{0}^{\theta^{*}}(1-\cos \theta)^{\gamma+\frac{n-1}{2}} f(\theta)(F(\theta))^{p-1} d \theta .
$$

Using the Hölder inequality, we obtain

$$
J \leq C \int_{0}^{\theta^{*}}(1-\cos \theta)^{\gamma+\frac{n-1}{2} p}(\sin \theta)^{-(n-2)(p-1)}(f(\theta))^{p} d \theta
$$

and from (3.7), we prove (3.5).

Remark 3.3. By the same way, we can prove that, if $\gamma \in \mathbb{R}$, satisfy $\gamma+\frac{1}{2}>0$ and $\left.\theta^{*} \in\right] 0, \pi / 2[$, then for any positive measurable function $f$ defined on $]-\theta^{*}, 0[$, such that

$$
\int_{-\theta^{*}}^{0}(1-\cos \theta)^{\gamma+\frac{p}{2}}[f(\theta)]^{p} d \theta<+\infty
$$


one has

$$
\int_{-\theta^{*}}^{0}(1-\cos \theta)^{\gamma}[F(\theta)]^{p} d \theta \leq C \int_{-\theta^{*}}^{0}(1-\cos \theta)^{\gamma+\frac{p}{2}}[f(\theta)]^{p} d \theta
$$

with

$$
F(\theta)=\int_{-\theta^{*}}^{\theta} f(t) d t
$$

Remark 3.4. (i) As a consequence of Inequality (3.5) for $n=2$ and Inequality (3.8), for any $w \in \mathcal{D}(]-\theta^{*}, \theta^{*}[)$ with $\gamma+\frac{1}{2}>0$, one has

$$
\int_{-\theta^{*}}^{\theta^{*}}(1-\cos \theta)^{\gamma}|w(\theta)|^{p} d \theta \leq C \int_{-\theta^{*}}^{\theta^{*}}(1-\cos \theta)^{\gamma+\frac{p}{2}}\left|w^{\prime}(\theta)\right|^{p} d \theta .
$$

(ii) Inequality (3.5) also implies that for any $w \in \mathcal{D}\left(\left[0, \theta^{*}[), \gamma+\frac{n-1}{2}>0\right.\right.$, one has

$$
\int_{0}^{\theta^{*}}(1-\cos \theta)^{\gamma}(\sin \theta)^{n-2}|w(\theta)|^{p} d \theta \leq C \int_{0}^{\theta^{*}}(1-\cos \theta)^{\gamma+\frac{p}{2}}(\sin \theta)^{n-2}\left|w^{\prime}(\theta)\right|^{p} d \theta .
$$

We now consider the sector

$$
S=S_{R, \lambda}=\left\{\mathbf{x} \in \mathbb{R}^{n}, r>R, 0<s<\lambda r\right\} \text {, with } R>0 \text { and } 0<\lambda<1 .
$$

We start to prove a Hardy-type inequality in the sector S.

Lemma 3.5. Let $\alpha, \beta \in \mathbb{R}$ such that $\beta>\max (0,(1-n+p) / 2 p)$. Then we have

$$
\forall u \in \mathcal{D}(S), \quad\|u\|_{L_{\alpha-\frac{1}{2}, \beta-\frac{1}{2}}^{p}(S)} \leq C\|\nabla u\|_{\mathbf{L}_{\alpha, \beta}^{p}(S)} .
$$

Proof. Let $u$ be in $\mathcal{D}(S)$. Since $\beta>0$, it is enough to prove

$$
I=\int_{S}(1+r)^{\left(\alpha-\frac{1}{2}\right) p} s^{\left(\beta-\frac{1}{2}\right) p}|u|^{p} d \mathbf{x} \leq C \int_{S}(1+r)^{\alpha p} s^{\beta p}|\nabla u|^{p} d \mathbf{x} .
$$

Indeed, let us assume that Inequality (3.13) holds. Then, if $0<\beta<\frac{1}{2}$, thanks to (3.13), we have

$$
\begin{aligned}
\int_{S}(1+r)^{\left(\alpha-\frac{1}{2}\right) p}(1+s)^{\left(\beta-\frac{1}{2}\right) p}|u|^{p} d \mathbf{x} & \leq \int_{S}(1+r)^{\left(\alpha-\frac{1}{2}\right) p} s^{\left(\beta-\frac{1}{2}\right) p}|u|^{p} d \mathbf{x} \\
& \leq C \int_{S}(1+r)^{\alpha p} s^{\beta p}|\nabla u|^{p} d \mathbf{x} \\
& \leq C \int_{S}(1+r)^{\alpha p}(1+s)^{\beta p}|\nabla u|^{p} d \mathbf{x} .
\end{aligned}
$$


Now, if $\beta \geq \frac{1}{2}$,

$$
\begin{aligned}
\int_{S}(1+r)^{\left(\alpha-\frac{1}{2}\right) p}(1+s)^{\left(\beta-\frac{1}{2}\right) p}|u|^{p} d \mathbf{x} & \leq C \int_{S}(1+r)^{\left(\alpha-\frac{1}{2}\right) p}\left(1+s^{\left(\beta-\frac{1}{2}\right) p}\right)|u|^{p} d \mathbf{x} \\
& \leq C \int_{S}(1+r)^{\alpha p}\left(s^{p / 2}+s^{\beta p}\right)|\nabla u|^{p} d \mathbf{x}
\end{aligned}
$$

and we obtain (3.12). First, we prove Inequality (3.13) for the case $n \geq 3$. Let $\left.\theta=\left(\theta_{1}, \theta_{2}, \ldots, \theta_{n-1}\right) \in\right] 0, \pi\left[{ }^{n-2} \times\right] 0,2 \pi\left[, R>0, \theta^{*} \in\right] 0, \frac{\pi}{2}[$ fixed and consider

$$
\Delta=\left\{(r, \theta) \in \mathbb{R}^{+} \times\right] 0, \pi\left[^{n-2} \times\right] 0,2 \pi\left[, r>R, \theta_{1} \in\right] 0, \theta^{*}[\} .
$$

To establish (3.13), we introduce the generalized spherical coordinates

$$
\begin{aligned}
& x_{1}=r \cos \theta_{1}, x_{2}=r \sin \theta_{1} \cos \theta_{2}, \ldots, x_{n-1}=r \sin \theta_{1} \ldots \sin \theta_{n-2} \cos \theta_{n-1}, \\
& x_{n}=r \sin \theta_{1} \ldots \sin \theta_{n-2} \sin \theta_{n-1},
\end{aligned}
$$

where $(r, \theta) \in \Delta$. Now taking $u(\mathbf{x})=v(r, \theta)$ and observing that

$$
\left|\frac{\partial v}{\partial \theta_{1}}\right| \leq r|\nabla u|
$$

it is sufficient to prove that

$$
\begin{aligned}
I & =\int_{\Delta}(1+r)^{\left(\alpha-\frac{1}{2}\right) p}\left(r-r \cos \theta_{1}\right)^{\left(\beta-\frac{1}{2}\right) p} r^{n-1}\left(\sin \theta_{1}\right)^{n-2}|v|^{p} d r d \theta \\
& \leq C \int_{\Delta}(1+r)^{\alpha p}\left(r-r \cos \theta_{1}\right)^{\beta p} r^{n-1}\left(\sin \theta_{1}\right)^{n-2} r^{-p}\left|\frac{\partial v}{\partial \theta_{1}}\right|^{p} d r d \theta
\end{aligned}
$$

We immediately have

$$
I \leq \int_{\Delta}(1+r)^{\alpha p} r^{\beta p}\left(1-\cos \theta_{1}\right)^{\left(\beta-\frac{1}{2}\right) p} r^{n-1}\left(\sin \theta_{1}\right)^{n-2} r^{-p}|v|^{p} d r d \theta .
$$

We now set

$$
J=\int_{0}^{\theta^{*}}\left(1-\cos \theta_{1}\right)^{\left(\beta-\frac{1}{2}\right) p}\left(\sin \theta_{1}\right)^{n-2}|v|^{p} d \theta_{1} .
$$

Since $\beta>(1-n+p) / 2 p$, we have $\left(\beta-\frac{1}{2}\right) p+\frac{n-1}{2}>0$. Moreover $u \in \mathcal{D}(S)$ implies that, for $(r, \theta) \in \Delta$, the function $\theta_{1} \rightarrow v(r, \theta)$ belongs to $\mathcal{D}\left(\left[0, \theta^{*}[)\right.\right.$. Therefore from (3.10), we get

$$
J \leq C \int_{0}^{\theta^{*}}\left(1-\cos \theta_{1}\right)^{\beta p}\left(\sin \theta_{1}\right)^{n-2}\left|\frac{\partial v}{\partial \theta_{1}}\right|^{p} d \theta_{1} .
$$

In view of inequalities (3.17) and (3.18), we obtain (3.16).

We now continue the proof of (3.13) for the case $n=2$. We define

$$
\Delta=\left\{(r, \theta) \in \mathbb{R}^{+} \times\right]-\pi, \pi[, r>R, \theta \in]-\theta^{*}, \theta^{*}[\}
$$

and we introduce the polar coordinates

$$
x_{1}=r \cos \theta, x_{2}=r \sin \theta,
$$


where $(r, \theta) \in \Delta$. Taking $u(\mathbf{x})=v(r, \theta)$, it is sufficient to prove

$$
\begin{aligned}
I & =\int_{-\theta^{*}}^{\theta^{*}} \int_{R}^{\infty}(1+r)^{\left(\alpha-\frac{1}{2}\right) p}(r-r \cos \theta)^{\left(\beta-\frac{1}{2}\right) p} r^{2}|v|^{p} d r d \theta \\
& \leq C \int_{-\theta^{*}}^{\theta^{*}} \int_{R}^{\infty}(1+r)^{\alpha p}(r-r \cos \theta)^{\beta p} r^{2-p}\left|\frac{\partial v}{\partial \theta}\right|^{p} d r d \theta .
\end{aligned}
$$

Proceeding as for the case $n \geq 3$ and the use of Inequality (3.9) give us Inequality (3.21).

Let $R$ be a positive real number fixed large enough. In the sequel, we will need the following Hardy-type inequality ( $c f$. Hardy-Littlewood-Polya [12] : we have

$$
\forall f \in \mathcal{D}(] R, \infty[), \quad \int_{R}^{+\infty}|f(r)|^{p} r^{\gamma} d r \leq C \int_{R}^{+\infty}\left|f^{\prime}(r)\right|^{p} r^{\gamma+p} d r, \quad \text { with } \gamma+1 \neq 0 .
$$

Let now $B_{R}$ denotes the open ball centered at the origin and with radius $R$ and $B_{R}^{\prime}=\mathbb{R}^{n} \backslash \bar{B}_{R}$. We are going to prove Inequality (3.12) for a function $u \in \mathcal{D}\left(B_{R}^{\prime}\right)$.

Lemma 3.6. Let $\alpha, \beta \in \mathbb{R}$ satisfy $\beta>\max (0,(1-n+p) / 2 p)$ and $\alpha+\beta+n / p-1 \neq$ 0 .Then, for any large enough positive real number $R$, we have

$$
\forall u \in \mathcal{D}\left(B_{R}^{\prime}\right), \quad\|u\|_{L_{\alpha-\frac{1}{2}, \beta-\frac{1}{2}}^{p}\left(B_{R}^{\prime}\right)} \leq C\|\nabla u\|_{\mathbf{L}_{\alpha, \beta}^{p}\left(B_{R}^{\prime}\right)} .
$$

Proof. Let $u$ be in $\mathcal{D}\left(B_{R}^{\prime}\right)$. We introduce the open set

$$
D_{R, \lambda}=\left\{\mathbf{x} \in \mathbb{R}^{n}, r>R, \lambda r<s\right\}
$$

and the following partition of unity

$$
\varphi_{1}, \varphi_{2} \in \mathcal{C}^{\infty}\left(B_{R}^{\prime}\right), \quad 0 \leq \varphi_{1}, \varphi_{2} \leq 1, \varphi_{1}+\varphi_{2}=1 \text { in } B_{R}^{\prime},
$$

with

$$
\begin{array}{r}
\varphi_{1}=1 \text { in } S_{R, \lambda / 2}, \quad \operatorname{supp} \varphi_{1} \subset S_{R, \lambda} \\
\text { and }\left|\nabla \varphi_{1}(\mathbf{x})\right| \leq \frac{C}{|\mathbf{x}|}, \mathbf{x} \in S_{R, \lambda} \cap D_{R, \lambda / 2} .
\end{array}
$$

We have

$$
\|u\|_{L_{\alpha-\frac{1}{2}, \beta-\frac{1}{2}}^{p}\left(B_{R}^{\prime}\right)} \leq\left\|u \varphi_{1}\right\|_{L_{\alpha-\frac{1}{2}, \beta-\frac{1}{2}}^{p}\left(B_{R}^{\prime}\right)}+\left\|u \varphi_{2}\right\|_{L_{\alpha-\frac{1}{2}, \beta-\frac{1}{2}}^{p}\left(B_{R}^{\prime}\right)} .
$$

Let us prove that

$$
\left\|u \varphi_{1}\right\|_{L_{\alpha-\frac{1}{2}, \beta-\frac{1}{2}}^{p}\left(B_{R}^{\prime}\right)} \leq C\|\nabla u\|_{\mathbf{L}_{\alpha, \beta}^{p}\left(B_{R}^{\prime}\right)} .
$$

Since $u \varphi_{1} \in \mathcal{D}\left(S_{R, \lambda}\right)$ and $\beta>\max (0,(1-n+p) / 2 p)$, Lemma 3.5 yields

$$
\left\|u \varphi_{1}\right\|_{L_{\alpha-\frac{1}{2}, \beta-\frac{1}{2}}^{p}}\left(S_{R, \lambda}\right) \leq C\left\|\nabla\left(u \varphi_{1}\right)\right\|_{\mathbf{L}_{\alpha, \beta}^{p}\left(S_{R, \lambda}\right)} .
$$


Furthermore, we have

$$
\begin{aligned}
\left\|\nabla\left(u \varphi_{1}\right)\right\|_{\mathbf{L}_{\alpha, \beta}^{p}\left(S_{R, \lambda}\right)}^{p} & \leq C \int_{S_{R, \lambda}}(1+r)^{\alpha p}(1+s)^{\beta p}|\nabla u|^{p} d \mathbf{x} \\
& +C \int_{S_{R, \lambda} \cap D_{R, \lambda / 2}}(1+r)^{\alpha p}(1+s)^{\beta p}\left|u \nabla \varphi_{1}\right|^{p} d \mathbf{x} .
\end{aligned}
$$

Since $s \sim r$ in $S_{R, \lambda} \cap D_{R, \lambda / 2}$ and from (3.24), for the second term of the right hand side of (3.27), we find

$$
\begin{aligned}
\int_{S_{R, \lambda} \cap D_{R, \lambda / 2}}(1+r)^{\alpha p}(1+s)^{\beta p}\left|u \nabla \varphi_{1}\right|^{p} d \mathbf{x} & \leq C \int_{S_{R, \lambda} \cap D_{R, \lambda / 2}}(1+r)^{(\alpha+\beta-1) p}|u|^{p} d \mathbf{x} \\
& \leq C \int_{S_{R, \lambda} \cap D_{R, \lambda / 2}} r^{(\alpha+\beta-1) p}|u|^{p} d \mathbf{x} .
\end{aligned}
$$

Now, we introduce the generalized spherical coordinates defined by (3.15), where $\left.(r, \theta) \in \mathbb{R}^{+} \times\right] 0, \pi\left[^{n-2} \times\right] 0,2 \pi[$, for the case $n \geq 3$, or the polar coordinates defined by $(3.20)$, where $\left.(r, \theta) \in \mathbb{R}^{+} \times\right]-\pi, \pi[$, for the case $n=2$. We take $u(\mathbf{x})=v(r, \theta)$ and, recalling that $\alpha+\beta+n / p-1 \neq 0$, we apply (3.22) to the function $r \rightarrow v(r, \theta)$. Thus, it comes

$$
\int_{R}^{+\infty}|v|^{p} r^{(\alpha+\beta-1) p+n-1} d r \leq C \int_{R}^{+\infty}\left|\frac{\partial v}{\partial r}\right|^{p} r^{(\alpha+\beta) p+n-1} d r
$$

which immediately yields

$$
\begin{aligned}
\int_{S_{R, \lambda} \cap D_{R, \lambda / 2}} r^{(\alpha+\beta-1) p}|u|^{p} d \mathbf{x} & \leq C \int_{S_{R, \lambda} \cap D_{R, \lambda / 2}} r^{(\alpha+\beta) p}|\nabla u|^{p} d \mathbf{x} \\
& \leq C \int_{S_{R, \lambda} \cap D_{R, \lambda / 2}}(1+r)^{\alpha p}(1+s)^{\beta p}|\nabla u|^{p} d \mathbf{x} .
\end{aligned}
$$

Summarizing (3.26), (3.27), (3.28) and (3.29), we deduce (3.25). Let us now prove that

$$
\left\|u \varphi_{2}\right\|_{L_{\alpha-\frac{1}{2}, \beta-\frac{1}{2}}^{p}\left(B_{R}^{\prime}\right)} \leq C\|\nabla u\|_{\mathbf{L}_{\alpha, \beta}^{p}\left(B_{R}^{\prime}\right)} .
$$

Since the support of $\varphi_{2}$ is included in $D_{R, \lambda / 2}$ and $\varphi_{2} \leq 1$, we have

$$
\begin{aligned}
\left\|u \varphi_{2}\right\|_{L_{\alpha-\frac{1}{2}, \beta-\frac{1}{2}}^{p}\left(B_{R}^{\prime}\right)}^{p} & =\int_{D_{R, \lambda / 2}}(1+r)^{\left(\alpha-\frac{1}{2}\right) p}(1+s)^{\left(\beta-\frac{1}{2}\right) p}\left|u \varphi_{2}\right|^{p} d \mathbf{x} \\
& \leq \int_{D_{R, \lambda / 2}}(1+r)^{\left(\alpha-\frac{1}{2}\right) p}(1+s)^{\left(\beta-\frac{1}{2}\right) p}|u|^{p} d \mathbf{x} .
\end{aligned}
$$

Moreover, recalling that $s \sim r$ in $D_{R, \lambda / 2}$, we get

$$
\int_{D_{R, \lambda / 2}}(1+r)^{\left(\alpha-\frac{1}{2}\right) p}(1+s)^{\left(\beta-\frac{1}{2}\right) p}|u|^{p} d \mathbf{x} \leq C \int_{D_{R, \lambda / 2}} r^{(\alpha+\beta-1) p}|u|^{p} d \mathbf{x} .
$$


Next, we use generalized spherical coordinates for $n \geq 3$ or polar coordinates for $n=2$, with $u(\mathbf{x})=v(r, \theta)$. Since $\alpha+\beta+n / p-1 \neq 0$, Inequality (3.22) yields

which implies that

$$
\int_{R}^{+\infty} r^{(\alpha+\beta-1) p+n-1}|v|^{p} d r \leq C \int_{R}^{+\infty} r^{(\alpha+\beta) p+n-1}\left|\frac{\partial v}{\partial r}\right|^{p} d r
$$

$$
\begin{aligned}
\int_{D_{R, \lambda / 2}} r^{(\alpha+\beta-1) p}|u|^{p} d \mathbf{x} & \leq C \int_{D_{R, \lambda / 2}} r^{(\alpha+\beta) p}|\nabla u|^{p} d \mathbf{x} \\
& \leq C \int_{D_{R, \lambda / 2}}(1+r)^{\alpha p}(1+s)^{\beta p}|\nabla u|^{p} d \mathbf{x} .
\end{aligned}
$$

The previous inequalities yield (3.30) and that concludes the proof.

We are now in a position to give the following Hardy-type inequality.

Theorem 3.7. Let $\alpha, \beta \in \mathbb{R}$ satisfy $\beta>\max (0,(1-n+p) / 2 p)$ and $\alpha+\beta+n / p-1 \neq$ 0 . Let $j^{\prime}=\min (j, 0)$, where $j$ is the highest degree of the polynomials contained in $X_{\alpha, \beta}^{1, p}\left(\mathbb{R}^{3}\right)$. Then, we have

$$
\forall u \in X_{\alpha, \beta}^{1, p}\left(\mathbb{R}^{3}\right), \quad \inf _{\lambda \in \mathbb{P}_{j^{\prime}}}\|u+\lambda\|_{L_{\alpha-\frac{1}{2}, \beta-\frac{1}{2}}^{p}}\left(\mathbb{R}^{n}\right) \leq C\|\nabla u\|_{\mathbf{L}_{\alpha, \beta}^{p}\left(\mathbb{R}^{n}\right)} .
$$

In other words, the semi-norm $|\cdot|_{X_{\alpha, \beta}^{1, p}\left(\mathbb{R}^{3}\right)}$ defines on $X_{\alpha, \beta}^{1, p}\left(\mathbb{R}^{3}\right) / \mathbb{P}_{j^{\prime}}$ a norm which is equivalent to the quotient norm.

Proof. The proof of this theorem is similar to that given in Amrouche-GiraultGiroire [2] (Theorem 8.3p 598).

Remark 3.8. Note that the particular case $n=3, p=2, \beta>0$ and $\alpha+\beta+\frac{1}{2}>0$ of previous theorem for was proved by Farwig (see [6]). Next, observe that the previous theorem also improves the inequalities proved in [13] (Lemma 2.3) for the case $n=3, p=2, \beta>0, \alpha \geq 0$ and $\alpha+\beta<\frac{3}{2}$.

Lemma 3.9. Let $\alpha, \beta$ be two reals such that $\beta \leq 0$ and $\alpha+n / p-1<0$ or $\alpha+\beta+$ $n / p-1>0$. Then, for any large enough positive real number $R$, we have

$$
\forall u \in \mathcal{D}\left(B_{R}^{\prime}\right), \quad\|u\|_{L_{\alpha-1, \beta}^{p}\left(B_{R}^{\prime}\right)} \leq C\|\nabla u\|_{\mathbf{L}_{\alpha, \beta}^{p}\left(B_{R}^{\prime}\right)} .
$$

Proof. Let $u \in \mathcal{D}\left(B_{R}^{\prime}\right)$. We first prove (3.32) for $n \geq 3$. Let $\theta=\left(\theta_{1}, \ldots, \theta_{n-1}\right)$ and consider the following set

$$
D=\left\{(r, \theta) \in \mathbb{R}^{+} \times\right] 0, \pi\left[{ }^{n-2} \times\right] 0,2 \pi[, r>R\} .
$$

We introduce the generalized spherical coordinates $(3.15)$ with $(r, \theta) \in D$. Taking $u(\mathbf{x})=v(r, \theta)$, Inequality (3.32) is equivalent to

$$
\begin{aligned}
I & =\int_{D} r^{(\alpha-1) p+n-1}\left(1+r-r \cos \theta_{1}\right)^{\beta p}\left(\sin \theta_{1}\right)^{n-2}|v|^{p} d r d \theta \\
& \leq C \int_{D} r^{\alpha p+n-1}\left(1+r-r \cos \theta_{1}\right)^{\beta p}\left(\sin \theta_{1}\right)^{n-2}\left|\frac{\partial v}{\partial r}\right|^{p} d r d \theta .
\end{aligned}
$$


We define $\tilde{r}\left(\theta_{1}\right)=\frac{1}{1-\cos \theta_{1}}$ and $\left.\tilde{\theta} \in\right] 0, \pi\left[\right.$ such that $R=\frac{1}{1-\cos \tilde{\theta}}$. We divide $D$ into three subdomains:

$$
\begin{aligned}
& D_{1}=\left\{(r, \theta) \in D, R<r<\tilde{r}\left(\theta_{1}\right), 0<\theta_{1}<\tilde{\theta}\right\} \text { where } 1+r-r \cos \theta_{1} \sim 1, \\
& D_{2}=\left\{(r, \theta) \in D, r>\tilde{r}\left(\theta_{1}\right), 0<\theta_{1}<\tilde{\theta}\right\} \text { where } 1+r-r \cos \theta_{1} \sim r-r \cos \theta_{1}, \\
& D_{3}=\left\{(r, \theta) \in D, r>R, \tilde{\theta}<\theta_{1}<\pi\right\} \text { where } 1+r-r \cos \theta_{1} \sim r-r \cos \theta_{1} .
\end{aligned}
$$

Thus, we obtain

$$
I \sim I_{1}+I_{2}+I_{3}
$$

with

$$
\begin{aligned}
I_{1} & =\int_{D_{1}} r^{(\alpha-1) p+n-1}\left(\sin \theta_{1}\right)^{n-2}|v|^{p} d r d \theta \\
I_{2} & =\int_{D_{2}} r^{(\alpha+\beta-1) p+n-1}\left(1-\cos \theta_{1}\right)^{\beta p}\left(\sin \theta_{1}\right)^{n-2}|v|^{p} d r d \theta \\
I_{3} & =\int_{D_{3}} r^{(\alpha+\beta-1) p+n-1}\left(1-\cos \theta_{1}\right)^{\beta p}\left(\sin \theta_{1}\right)^{n-2}|v|^{p} d r d \theta .
\end{aligned}
$$

Let us now estimate the three integrals. Since $\alpha+\frac{n}{p}-1 \neq 0$, an integration by parts and the Hölder's inequality yield

$$
\begin{aligned}
& \left.\int_{R}^{\tilde{r}\left(\theta_{1}\right)} r^{(\alpha-1) p+n-1}|v|^{p} d r \leq \frac{1}{(\alpha-1) p+n}\left(\tilde{r}\left(\theta_{1}\right)\right)^{(\alpha-1) p+n} \mid v\left(\tilde{r}\left(\theta_{1}\right), \theta\right)\right)\left.\right|^{p} \\
& +\frac{p}{|\alpha p-p+n|}\left(\int_{R}^{\tilde{r}\left(\theta_{1}\right)} r^{(\alpha-1) p+n-1}|v|^{p} d r\right)^{1 / p^{\prime}}\left(\int_{R}^{\tilde{r}\left(\theta_{1}\right)} r^{\alpha p+n-1}\left|\frac{\partial v}{\partial r}\right|^{p} d r\right)^{1 / p},
\end{aligned}
$$

and consequently

$$
\begin{aligned}
I_{1} & \leq \frac{1}{(\alpha-1) p+n} \int_{0}^{2 \pi} \int_{0}^{\pi} \ldots \int_{0}^{\pi} \int_{0}^{\tilde{\theta}}\left(\tilde{r}\left(\theta_{1}\right)\right)^{(\alpha-1) p+n}\left(\sin \theta_{1}\right)^{n-2}\left|v\left(\tilde{r}\left(\theta_{1}\right), \theta\right)\right|^{p} d \theta_{1} \ldots d \theta_{n-1} \\
& +C \int_{D_{1}} r^{\alpha p+n-1}\left(\sin \theta_{1}\right)^{n-2}\left|\frac{\partial v}{\partial r}\right|^{p} d r d \theta .
\end{aligned}
$$

Similarly, since $\alpha+\beta+\frac{n}{p}-1 \neq 0$, we get for the two other integrals

$$
\begin{aligned}
& I_{2} \leq-\frac{1}{(\alpha+\beta-1) p+n} I_{2}^{\prime} \\
& +C \int_{D_{2}} r^{(\alpha+\beta) p+n-1}\left(1-\cos \theta_{1}\right)^{\beta p}\left(\sin \theta_{1}\right)^{n-2}\left|\frac{\partial v}{\partial r}\right|^{p} d r d \theta,
\end{aligned}
$$

with

$$
I_{2}^{\prime}=\int_{0}^{2 \pi} \int_{0}^{\pi} \ldots \int_{0}^{\pi} \int_{0}^{\tilde{\theta}}\left(\tilde{r}\left(\theta_{1}\right)\right)^{(\alpha+\beta-1) p+n}\left(1-\cos \theta_{1}\right)^{\beta p}\left(\sin \theta_{1}\right)^{n-2}\left|v\left(\tilde{r}\left(\theta_{1}\right), \theta\right)\right|^{p} d \theta_{1} \ldots d \theta_{n-1},
$$




$$
I_{3} \leq C \int_{D_{3}} r^{(\alpha+\beta) p+n-1}\left(1-\cos \theta_{1}\right)^{\beta p}\left(\sin \theta_{1}\right)^{n-2}\left|\frac{\partial v}{\partial r}\right|^{p} d r d \theta .
$$

Summarizing (3.34), (3.35), (3.36), we obtain

$$
\begin{aligned}
& I \leq C \int_{D} r^{\alpha p+n-1}\left(1+r-r \cos \theta_{1}\right)^{\beta p}\left(\sin \theta_{1}\right)^{n-2}\left|\frac{\partial v}{\partial r}\right|^{p} d r d \theta \\
& +\frac{1}{(\alpha-1) p+n} \int_{0}^{2 \pi} \int_{0}^{\pi} \ldots \int_{0}^{\pi} \int_{0}^{\tilde{\theta}}\left(\tilde{r}\left(\theta_{1}\right)\right)^{(\alpha-1) p+n}\left(\sin \theta_{1}\right)^{n-2}\left|v\left(\tilde{r}\left(\theta_{1}\right), \theta\right)\right|^{p} d \theta_{1} \ldots d \theta_{n-1} \\
& -\frac{1}{(\alpha+\beta-1) p+n} I_{2}^{\prime} .
\end{aligned}
$$

Recalling that $\left(\tilde{r}\left(\theta_{1}\right)\left(1-\cos \theta_{1}\right)\right)^{\beta p}=1$, and since $\beta \leq 0$ with $\alpha+\frac{n}{p}-1<0$ or $\alpha+\beta+\frac{n}{p}-1>0$, we have

$$
\frac{1}{(\alpha-1) p+n}-\frac{1}{(\alpha+\beta-1) p+n} \leq 0 .
$$

Thus, we deduce Inequality (3.33).

For the case $n=2$, we use polar coordinates $(3.20)$, with $(r, \theta) \in D$, where

$$
D=\left\{(r, \theta) \in \mathbb{R}^{+} \times\right]-\pi, \pi[, r>R\} .
$$

We set $\tilde{r}(\theta)=\frac{1}{1-\cos \theta}$ and $\tilde{\theta}$ such that $R=\frac{1}{1-\cos \tilde{\theta}}$. We devide $D$ as follow

$$
\begin{aligned}
& D_{1}=\{(r, \theta) \in D, R<r<\tilde{r}(\theta),-\tilde{\theta}<\theta<\tilde{\theta}\} \\
& D_{2}=\left\{(r, \theta) \in D, r>\tilde{r}(\theta),-\tilde{\theta}<\theta_{1}<\tilde{\theta}\right\} \\
& D_{3}=\{(r, \theta) \in D, r>R, \theta \in]-\pi,-\tilde{\theta}[\cup] \tilde{\theta}, \pi[\} .
\end{aligned}
$$

We then proceed as for the proof of the case $n \geq 3$.

Now proceeding as for the case $\beta>0$, we obtain the Hardy-type inequality.

Theorem 3.10. Let $\alpha, \beta$ be two real satisfying $\beta \leq 0$ and $\alpha+\frac{n}{p}-1<0$ or $\alpha+\beta+\frac{n}{p}-1>0$. Let $j^{\prime}=\min (j, 0)$, where $j$ is the highest degree of the polynomials contained in $Y_{\alpha, \beta}^{1, p}\left(\mathbb{R}^{3}\right)$. Then we have

$$
\forall u \in Y_{\alpha, \beta}^{1, p}\left(\mathbb{R}^{3}\right), \quad \inf _{\lambda \in \mathbb{P}_{j^{\prime}}}\|u+\lambda\|_{L_{\alpha-1, \beta}^{p}\left(\mathbb{R}^{n}\right)} \leq C\|\nabla u\|_{\mathbf{L}_{\alpha, \beta}^{p}\left(\mathbb{R}^{n}\right)} .
$$

In other words, the semi-norm $|\cdot|_{Y_{\alpha, \beta}^{1, p}\left(\mathbb{R}^{3}\right)}$ defines on $Y_{\alpha, \beta}^{1, p}\left(\mathbb{R}^{3}\right) / \mathbb{P}_{j^{\prime}}$ a norm which is equivalent to the quotient norm. 
Remark 3.11. For the case $\beta=0$, we get the result proved by Amrouche-GiraultGiroire [2] for the space $W_{\alpha}^{1, p}\left(\mathbb{R}^{n}\right)$ when $\alpha+\frac{n}{p}-1 \neq 0$.

\section{References}

[1] C. Amrouche and H. Bouzit, $L^{p}$-Inequalities for Scalar Oseen Potential. J. Math. Anal. Appl., 337 (2008), 753-770.

[2] C. Amrouche, V. Girault and J. Giroire, Weighted Sobolev spaces for Laplace's equation in $\mathbb{R}^{n}$. J. Math. Pures. Appl. 73 (1994), 579-606.

[3] C. Amrouche and U. Razafison, Weighted Sobolev Spaces for a Scalar Model of the Stationary Oseen Equations in $\mathbb{R}^{3}$. J. Math. Fluid Mech. 9 (2007), 181-210.

[4] C. Amrouche and U. Razafison, The Stationary Oseen Equations in $\mathbb{R}^{3}$. An approach in Weighted Sobolev Spaces. J. Math. Fluid Mech. 9 (2007), 211-225.

[5] C. Amrouche and U. Razafison, Espaces de Sobolev avec poids et équation scalaire d'Oseen dans $\mathbb{R}^{n}$. C. R. Math. Acad. Sci. Paris. 337(12) (2003), 761-766.

[6] R. Farwig, A variational approach in weighted Sobolev spaces to the operator $-\Delta+$ $\partial / \partial x_{1}$ in exterior domains of $\mathbb{R}^{3}$. Math. Z. 210(3) (1992), 449-464.

[7] R. Farwig, The stationary exterior 3D-problem of Oseen and Navier-Stokes equations in anisotropically weighted Sobolev spaces. Math. Z. 211(3) (1992), 409-447.

[8] R. Farwig and H, Sohr, Weighted estimates for the Oseen equations and the NavierStokes equations in exterior domains. In: Ser. Adv. Math. Appl. Sci. 47 (1998), 11-30.

[9] R. Finn, On the exterior stationary problem for the Navier-Stokes equations, and associated perturbation problems. Arch. Rational Mech. Anal. 19 (1965), 363-406.

[10] R. Finn, Estimates at infinity for stationary solutions of the Navier-Stokes equations. Bull. Math. Soc. Sci. Math. Phys. R. P. Roumaine 3(51) (1959), 387-418.

[11] G. P. Galdi, An introduction to the mathematical theory of the Navier-Stokes equations. Vol. I, , Springer Tracts in Natural Philosophy, 38, Springer-Verlag, 1994.

[12] G. H. Hardy, J. E. Littlewood and G. Pólya, Inequalities. Cambridge, at the University Press, 1952.

[13] S. Kračmar, Š. Nečasová, P. Penel, Anisotropic L2 ${ }^{2}$-estimates of weak solutions to the stationary Oseen type equations in $\mathbb{R}^{3}$ for rotating body. RIMS Kokyuroku Bessatsu, B1 (2007), 219-235.

[14] S. Kračmar, A. Novotný and M. Pokorný, Estimates of Oseen kernels in weighted $L^{p}$ spaces. J. Math. Soc. Japan 53 (2001), 59-111.

[15] P. I. Lizorkin, $\left(L^{p}, L^{q}\right)$-multipliers of Fourier integrals. Dokl. Akad. Nauk SSSR 152, (1963), 808-811.

[16] B. Muckenhoupt, Hardy's inequality with weights. Studia Math. 44 (1972), 31-38.

[17] C. W. Oseen, Uber die Stokessesche Formel und Über eine Verwandte Aufgabe inder Hydrodynamik., Journal Ark. Mat. Astron. Fys. 29(6) (1910), 1-20.

[18] C. W. Oseen, Neuere Methoden und Ergebnisse in der Hydrodynamik. Akadem. Verlagsgesellschaft, Leipzig, 1927.

[19] M. Reed and B. Simon, Fourier Analysis Self-Adjointness. t. II, Academic Press, (1975). 
[20] E. M. Stein, Singulars Integrals and Differentiability Properties of Functions. Princeton New Jersey, 1970.

Chérif Amrouche

Laboratoire de Mathématiques Appliquées

UMR CNRS 5142

Université de Pau et des Pays de l'Adour, IPRA,

Avenue de l'Université, 64000 Pau, France.

e-mail: cherif .amrouche@univ-pau.fr

Ulrich Razafison

MAPMO, UMR CNRS 6628,

Fédération Denis Poisson,

bât. Mathématiques,

B. P. 6759, Orléans, Cedex 2, France.

e-mail: ulrich.razafison@math.cnrs.fr 